\title{
Global Standardization of Surgical Training
}

\author{
Vijay K. Mittal
}

Published online: 29 October 2014

(C) Association of Surgeons of India 2014

The continuing requirement of highly skilled surgeons relies on a good surgical training system. It is important that the global development of surgical training programs be set up to have standardized education and training to produce competent and skilled surgeons globally. To standardize surgical education first, it is imperative to identify the factors that need improvement in other countries in comparison to the US training system. In order to achieve this objective, it is important to focus on a developing country's training system to evaluate the changes required. Since many Indian physicians immigrate to the USA seeking to practice medicine, India was chosen to be the focus of a detailed evaluation from different perspectives. For evaluating the Indian surgical training system critically from different perspectives, it was important to involve the institutions, staff, as well as the residents. On-site observations and surveys of the staff and the residents were planned. The survey was designed to examine the surgical education and training of residents, to delineate the current situation, and to discuss the possible reform and future development of surgical training systems, in order to put forward solutions and countermeasures. The comparison of the results was made to the US training system as a reference to achieve a level of parity globally, thus standardizing the surgical education and training in India.

The field of surgery is an ever evolving one, and there is a need for a system that is acceptable and dynamic to keep pace with the rapidly changing knowledge base and technology. Teaching, training, and evaluation of general surgery residents in India have not been reviewed lately. In 2001, the Medical

V. K. Mittal $(\square)$

Department of Surgery, Providence Hospital \& Medical Centers, Southfield, MI, USA

e-mail: vijay.mittal@stjohn.org

V. K. Mittal

Wayne State University, School of Medicine, Detroit, MI, USA
Council of India (MCI) recommended 3 years of in-house training imparting surgical skills, the ability to collect and analyze data, dissertation writing and theory examination on the content and clinical examination to assess the abilities to recognize the signs and symptoms, and communication skills and manners for accrediting masters in general surgery all over the country [1]. The surgical training description by MCI appears adequate; however, actual implementation and quality control is not uniform across the country. Moreover, the surgical training offered is not recognized by the North American system, posing difficulties in transferring medical training and credentials earned to the North American system.

The recent emerging changes in health care delivery have a significant impact on the structure of surgical education in academic departments of surgery. This article encourages a proactive stance by surgical educators to anticipate changes and move toward restructuring in areas of curricular content, the teaching process, performance evaluation strategies, and faculty infrastructure of the academic department and use the advanced experience of foreign countries as references to carry out the policy of "Education must be modernized, catch up with the world's level, and be future oriented" [2]. In order to achieve the above goals, a proposal, titled "Global Surgical Education Evaluation and Uniformity" has been considered by Fulbright Commission, the pioneers of which will work with other surgical educators to enhance the country's surgical training curriculum to bring it in line with that of the USA.

To achieve the above goals and targets, the Providence Hospital Surgery Department conducted a survey of institutions in India during March 2013-March 2014 to determine current standards of surgical education being offered to surgical residents. On-site observations and separate questionnaire surveys were conducted from institutional authorities as well as residents. The survey was formulated and distributed among institutional authorities and residents. The questionnaire was divided into five building blocks focusing on 
different aspects of the standards of surgical education. Results were analyzed to discover standards of surgical education.

On the basis of the survey, general surgery training in India needs to be improved and revised. The revised training should be overseen by a third party, thus creating uniformity in surgical residency training. Broad-based standardized training patterns during surgical residency in theoretical knowledge, surgical skills, and research methodology should be a part of a revised surgical program along with multipoint evaluation and centralized examination. Emphasis on thesis writing and research publications should be put forward. In order to bring general surgery training in India up to par with the Western world, relocation of resources is a must. This will put the emphasis on the international exchange of surgeons, thereby limiting the barrier for the mobility of qualified Indian surgeons. Criterion for the minimum number of surgeries in which trainees should assist senior surgeons and also senior surgeons to assist the trainee should be established. Guidelines for the duty hours and/or work-hour restrictions should be passed and uniformly followed. The quality of faculty needs to be improved.

As per the survey, no emphasis has been placed on the use of modern technology in improving surgical skills in residents. There is increasing evidence provided by surgical laboratories that the use of simulators and trainers improves the operating room performances. Mandatory courses like Advanced Cardiac Life Support, Advanced Trauma Life Support, Pediatric Advanced Life Support, and Fundamentals of Laparoscopic Surgery, and Fundamentals of Endoscopic Surgery should be introduced into surgery residency programs. Training programs in robotic surgery need to be formally established in residency programs. In addition, laparoscopic skills should also be taught and incorporated into training protocols.

The evaluation and examination system of present surgery residency programs need to be reevaluated. A national organization similar to the US American Board of Surgery should be established, which will centralize evaluation and certification of surgical education, such as the Masters in Surgery and Diploma of National Board exams. Yearly in-service exams and stringent feedback from the staff, faculty, and peers should be established. Feedback mechanisms regarding resident concerns and appropriate remediation protocols to guide underperforming residents should be established. Research facilities should be provided and residents should be encouraged to conduct research. From the survey, it is evident that the residents want to pursue additional training and fellowship in USA and want to be certified by the American Board of Surgeons. Guidelines should be made for international rotations and electives, which will be useful to both Indian and North American educators to share processes and information. It will also create an interest in the exchange of residents and faculty members between the two countries.

The hectic traditional work schedule of the residents has often had negative influences on education and the quality of patient care, sometimes compromising the competence of the residents. Faculty supervision in emergency and operation is not consistent across the board, which should be addressed.

The acquisition of knowledge can be autonomous and selfdirected. However, postgraduate teaching by experienced surgeons forms a stronger foundation and goes a long way. The present system needs more experienced teachers with an academic mind set and more teaching hours per week. There should be a mix of supervision and independence for surgical residents in order to learn and grow.

Surgical education in independent India started in the 1950 s, when the MS surgery program was for 3 years followed by an evaluation at the end. This system has yet not undergone any major changes. Serious efforts need to be made in order to restructure the general surgery program, so as to bring general surgery up to the standards of the Western world. The duration of the formal training of surgeons should be increased and made uniform across India. In a peer review poll conducted in India, the majority of responders voted in favor of changes in the current general surgery postgraduate program [3].

Surgical education is now at a crucial point in India. Reform and development of the world's medical science and surgical education has had a profound influence on the surgical education, and these changes need to be implemented in India so as to achieve parity globally.

\section{References}

1. Medical Council of India (MCI), Post graduate medical education regulations 2000

2. Zhang WG (2005) Facilitating residency training in China with the medical education experiences of America. Med Educ (China) 1(2): 77-80

3. Gupta A, Kumar S, Mishra MC, Kumar S (2006) Surgical residency program: training, teaching and evaluation in general surgery - a peer opinion poll in five medical colleges in northern India. Indian J Surg 68:310-315 